\title{
LOS MAPAS CONCEPTUALES
}

Iván Giraldo Enciso

\section{ABSTRACT}

El presente trabajo busca dar a entender una herramienta versátil para el trabajo de los docentes dentro de sus aulas. En nuestra práctica docente hemos aplicado esta estrategia de enseñanza aprendizaje con buenos resultados, por lo que el maestro que la aplique tendrá logros evidentes en sus estudiantes.

La propuesta práctica nos evitará ahondar en los presupuestos teóricos sobre mapas conceptuales, que dicho sea de paso, ya han sido suficientemente probados y aceptados por la comunidad académica. Nuestra preocupación es, cómo aplicamos esta estrategia en nuestro quehacer cotidiano dentro de las aulas, por ello damos diversos consejos para aplicarlos en las aulas desde el nivel inicial, a través de los mapas nocionales, pasando por la primaria y culminando con la secundaria.

Además, se presentan las posibilidades de evaluar los diversos mapas conceptuales producidos en clase. Debemos recalcar que no hay mapa conceptual unívoco y que, quizás, la mejor evaluación se daría cuando el estudiante exponga o pueda resolver algunas preguntas, teniendo sus mapas conceptuales a la mano.

Al final, publicamos una tesina sobre los mapas conceptuales, que presentamos para graduarnos del diplomado internacional "Didáctica para un Aprendizaje Desarrollador y Creativo", desarrollado en Lima el año 2004, por la Derrama Magisterial, en convenio con el Instituto Pedagógico Latinoamericano y Caribeño (IPLAC-Cuba). 


\section{PRÓLOGO}

Nihil humanum alienum me est

Los mapas conceptuales, como estrategia de enseñanza aprendizaje, han tenido una vida muy ajetreada, por lo que podríamos hacer algunos comentarios al respecto: Se han planteado de diversas formas y, por lo tanto, se han malentendido sus principios. El mapa es "conceptual", no de frases, oraciones, ideas, sino de conceptos. Así, vemos una gran diversidad de los llamados "mapas conceptuales": mapas semánticos, árboles de conocimiento, no se siguen rigurosamente los principios básicos de los mapas conceptuales, que no son muchos y, además, son simples. Los hay de diversas formas: araña, jerárquico (vertical u horizontal): mapa araña, mapa organigrama, mapa sistémico, mapa multidimensional, mapa de paisaje, diagrama de flujo; ello se debe a la diversidad y creatividad de nuestros estudiantes. Por esta razón el maestro no debe hacerlo, sino los estudiantes, bajo nuestra dirección u orientación. Pues ellos tienden a "complacernos" en la elaboración de cualquier estrategia que planteemos y siguen a pie juntillas los ejemplos que damos, con la finalidad de conseguir una aprobación o buena calificación de sus trabajos.

Así, en un primer momento debemos ser "puristas" en su elaboración; una vez que veamos que nuestros estudiantes manejen adecuadamente los principios de estos, podemos ser más flexibles en su elaboración y calificación, pues, recordemos que todas las estrategias de enseñanza aprendizaje tienen por finalidad darles instrumentos para que puedan aprender a aprender, es decir que puedan aprender por ellos mismos. Lo que queremos decir es que, una vez que los estudiantes se vuelvan diestros en la elaboración de los mapas conceptuales podríamos darle libertad para que ellos, con su creatividad, puedan incorporar algunos otros elementos.

Por ello, el presente trabajo ahonda en la parte práctica, dando diversos consejos de cómo aplicarlos en las aulas desde el nivel inicial, a través de los mapas nocionales, pasando por los otros niveles de primaria y culminando con el de secundaria. Además, se presentan las posibilidades de evaluar y, quizás la mejor evaluación se daría cuando él exponga o pueda resolver algunas preguntas teniendo sus mapas conceptuales a la mano. 


\section{INTRODUCCIÓN}

La noción de mapas conceptuales se desarrolló en la década de los sesenta del siglo pasado, en el Departamento de Educación de la universidad norteamericana de Cornell.

Surge como una forma de instrumentalizar la teoría del aprendizaje significativo sostenida por Ausubel, sobre todo en lo referente a la evolución de las ideas previas que poseen los estudiantes.

Estas ideas fueron desarrolladas por un grupo de investigadores bajo la supervisión del Dr. Joseph D. Novak, por medio del programa denominado "Aprender a Aprender", en el que se pretende un objetivo fundamental: liberar el potencial de aprendizaje en los hombres que permanece sin desarrollar y que muchos programas educativos entorpecen, en vez de facilitarlos. Desde ese momento, se inicia todo un movimiento en busca de estrategias pedagógicas que favorezcan dicha práctica educativa.

\section{ARGUMENTOS TEÓRICOS}

Los mapas conceptuales permiten utilizar ambos hemisferios del cerebro, pues según las nuevas ciencias de la cognición, el proceso de organización espacial, así como la inteligencia intuitivo-emotiva, es propio del hemisferio derecho; mientras que la lectoescritura, el análisis léxico-conceptual, el raciocinio abstracto y las matemáticas, son propios del hemisferio izquierdo. De este modo, los procesos de pensamiento abstracto y los psicomotrices se complementan.

Está técnica se inscribe dentro de la concepción constructivista del aprendizaje, desarrollada por el Dr. Joseph D. Novak para desarrollar el aprendizaje significativo, que propicia la reorganización de los conocimientos por medios de esquemas o resúmenes.

Sin lugar a dudas, estas ideas se inician con los estudios de Piaget, que propone la existencia de esquemas conceptuales en la mente de todos los individuos.

Además, tenemos los planteamientos pedagógicos del ya citado Ausubel, que afirma la existencia de cuatro tipos de aprendizajes:

a) Repetitivo;

b) Por descubrimiento;

c) Mecánico o memorístico;

d) Significativo. 
Según Ausubel, el aprendizaje significativo se alcanza cuando el estudiante incorpora un nuevo aprendizaje dentro de su estructura cognitiva. Este aprendizaje integra, modifica, establece relaciones y coordina entre esquemas de conocimientos que ya posee el estudiante. Para lograr el aprendizaje significativo, nuestro autor considera que deben darse las siguientes condiciones dentro del aula:

a) El estudiante debe mostrar una actitud positiva hacia el llamado aprendizaje significativo, es decir, debe estar motivado.

b) El contenido de lo que se va a trabajar debe ser potencialmente significativo; esto quiere decir que los elementos sean claros, coherentes y bien organizados (significatividad lógica), y debe estar de acuerdo con el nivel de desarrollo y los conocimientos previos de los estudiantes (significatividad psicológica).

Por ello, Ausubel recomienda averiguar primero lo que el estudiante sabe, para que a partir de ello procedamos a impartir los nuevos conocimientos.

El aprendizaje significativo posee algunas características:

- Está centrado en el educando, que es el protagonista del aprendizaje.

- Busca su desarrollo integral, superando el memorismo.

- Incrementa la autoestima y la seguridad académica del educando.

Por todo ello, Novak considera que los mapas conceptuales son una herramienta de trabajo que permite la confrontación y el análisis de la forma de pensar de los estudiantes, brindando mejores oportunidades para el logro del aprendizaje significativo.

\section{Principio básico}

El principio básico que se da en la elaboración de los mapas conceptuales reside en la afirmación de que las ideas expresadas simbólicamente se van relacionando, coherentemente, con lo que el estudiante ya sabe.

Por ello decimos que los mapas conceptuales son representaciones gráficas de las relaciones entre conceptos, en forma de proposiciones, que establece el estudiante en su estructura cognitiva.

Cuando hemos trabajado adecuadamente los principios básicos de los mapas conceptuales, nuestros estudiantes, que han aprendido otros conocimientos, elaborarán un mapa conceptual que represente la relación conceptual en su mente; por lo tanto, el estudiante aprende a aprender, educándose a sí mismo, logrando controlar mejor los significados que conforman su vida. 


\section{Ventajas para el estudiante}

Los mapas conceptuales proporcionan las siguientes ventajas para el estudiante:

a) Construye su propio aprendizaje, haciéndolo responsable de su aprendizaje significativo, siendo él el protagonista principal.

b) Manejará su propio método, que le proporcionará una nueva forma de pensar y sentir.

c) Conoce sus debilidades y fortalezas.

d) Comprende mejor el mundo y busca cambiarlo.

e) Identifica los conceptos claves y sugiere conexiones coherentes entre lo nuevo y lo que ya posee.

f) Practica la responsabilidad y honradez intelectual.

g) Se respeta a sí mismo y a sus profesores.

\section{Cuidados}

Los profesores debemos estar atentos a los siguientes defectos:

a) Evitar diagramas de flujo donde se presenten secuencias lineales.

b) Evitar relaciones enredadas.

c) Eitar que sea la única herramienta o técnica para construir el aprendizaje.

d) Debemos de tener muy en cuenta que la construcción de mapas conceptuales es un proceso, como todo aprendizaje, para el que necesitamos tiempo, donde el estudiante practique el pensamiento reflexivo. No debemos caer en el inmediatismo.

e) No pensar que los mapas conceptuales son unívocos, por ello debemos de valorar los mapas conceptuales que hayan elaborado nuestros estudiantes, pues eso ya es un esfuerzo, que debe ser mejorado.

\section{Elementos de un mapa conceptual}

En la teoría de los mapas conceptuales, el aprendizaje de conceptos constituye el elemento central, ya que ellos establecen la estructura del conocimiento y constituyen la base para la construcción del significado.

Definimos concepto como las palabras que se emplean para designar la imagen de un objeto o acontecimiento que se genera en la mente de un individuo. Estas palabras pueden designar acontecimientos, objetos, animales, cualidades. 
Los mapas conceptuales tienen por objeto presentar relaciones significativas entre conceptos unidos por palabras, que llamaremos palabras-enlace, formando una unidad semántica 0 proposición. Entonces tendremos que las proposiciones son frases que están constituidas por dos o más conceptos unidos por una palabra enlace y que tienen sentido lógico.

Las palabras-enlace sirven para unir los conceptos formando proposiciones, están escritas sobre unas líneas que facilitan la visualización de las relaciones con los conceptos. Asimismo, las palabras enlace provocan imágenes mentales. Las palabras enlace pueden ser verbos, artículos, conjunciones, pero nunca conceptos. Así, estas palabras pueden ser distintas a las que aparecen en el texto, mientras mantenga el mismo significado de la proposición, por ello el estudiante puede inventar la palabra enlace más adecuada.

\section{Signos gráficos de los mapas conceptuales}

Los MCs contienen los siguientes signos gráficos:

- Elipses, en ellas se colocan los conceptos, poseen cualidades únicas que los psicólogos destacan, entre otros gráficos.

- Líneas, unen las elipses, junto a ellas se escriben las palabras enlace.

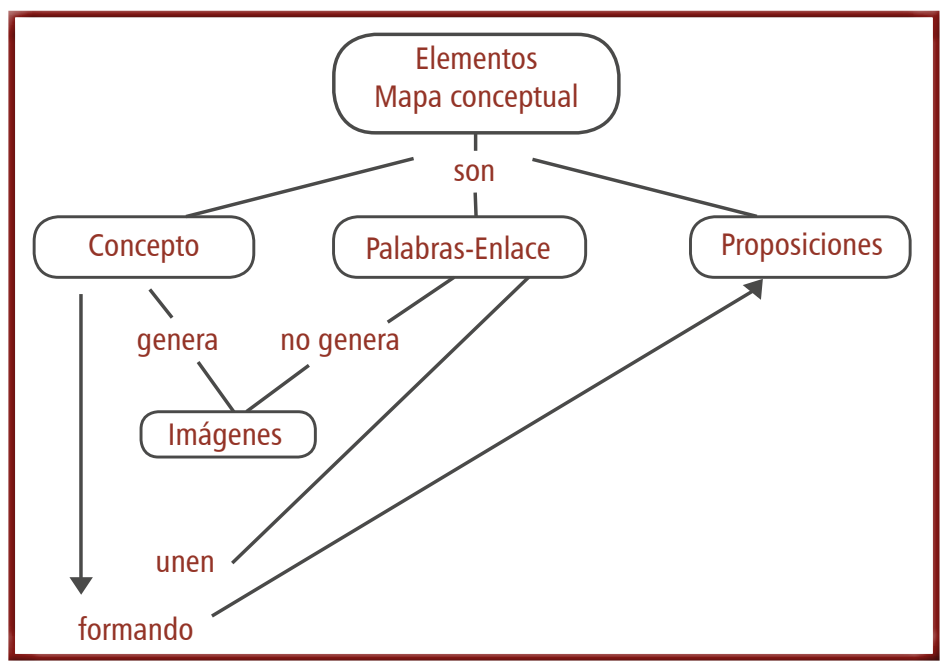

\section{Impacto visual}

El uso de elipses para los conceptos y las líneas para las palabras enlace dan lugar al impacto visual, que es muy provechoso para la organización de nuestras ideas. 
Debemos de tener en cuenta que los mapas conceptuales facilitan la memorización; por ello, sostenemos que la memoria es una función del cerebro que implica diversos procesos, entre ellos: selección, abstracción, interpretación, integración y recuperación.

\section{Aplicaciones}

Los mapas conceptuales tienen sus antecedentes en los resúmenes, síntesis, cuadros sinópticos y demás ayudas para el aprendizaje, que han sido estudiados explícitamente por las ciencias de la educación y las ciencias cognitivas actuales, encontrando gran expansión por medio de la tecnología educativa.

Novak utilizó los mapas conceptuales, en un primer momento, para las ciencias naturales; luego afirmó que se podían usar en las diversas áreas. Por su parte, Ontoria sustenta esta afirmación citando experiencias en distintas áreas como la matemática, las ciencias sociales y, por supuesto, las ciencias naturales.

\section{Características}

Hemos sostenido que los mapas conceptuales facilitan la memorización, ya que al elaborarlos se desarrollan los procesos mentales anteriormente anotados, pero los alcances de los mapas conceptuales van mucho más allá de las utilidades ya señaladas.

En líneas generales, los mapas conceptuales muestran tres características o condiciones que los diferencian de otras técnicas cognitivas:

a) Jerarquía: es importante para la elaboración del mapa conceptual que los conceptos estén dispuestos en orden de importancia de más generales o inclusivos a los de menor inclusividad.

Los especialistas nos dicen que un concepto debe aparecer solo una vez; sin embargo, esto debemos de aplicarlo de acuerdo a las circunstancias, en la medida de lo posible.

b) Selección: los mapas conceptuales se centran en los conceptos que se hallan dentro de un texto significativo. Esto nos puede llevar a llenarnos de conceptos. Por ello, con el afán de claridad, debemos de excluir algunos conceptos que se hallan implícitos en otros, o que se hallen subsumidos en otros. Por lo que debemos centrarnos en el proceso de selección de conceptos.

c) Impacto visual: las elipses y las líneas dan un impacto visual que serán provechosos para la organización de nuestras ideas. Veamos lo que nos dice Joseph Novak de ello: 
"Un buen mapa conceptual es conciso y muestra las relaciones entre las ideas principales de un modo simple y vistoso, aprovechando la notable capacidad humana para la representación visual"

\section{Concepto de mapa conceptual}

En conclusión, un mapa conceptual es un instrumento educativo o esquema que permite elaborar una representación de relaciones significativas y jerárquicas entre conceptos nuevos, con abstracciones generales ya adquiridas previamente, enfatizando las relaciones cruzadas entre grupos de conceptos, globalizando el conjunto de relaciones en una amplia construcción mental en forma de proposiciones.

Los mapas conceptuales son conocidos con diversos nombres lo que trae como consecuencia una gran confusión, entre otros tenemos:

- Mapas conceptuales (Novak, Gowin y Ontoria)

- Representaciones Estructuradas (Bachman y Levesque)

- Formas de pensamiento (Keegan)

- Formas epistemológicas (Collins y Ferguson)

- Esquemas abstractos (Ohlsson)

- Mapas mentales (Perkins)

- Árboles de conocimiento (Mioduser y Santamaría)

\section{Principios metodológicos para la elaboración de mapas conceptuales}

Una vez conocidos los elementos y signos gráficos, podemos definir los mapas conceptuales como un esquema gráfico, conformado por la unión de conceptos (encerrados en las elipses) por medio de líneas que ayudan a su visualización. Sobre estas líneas van las palabras-enlace que detallan el tipo de relación entre los conceptos formando, de esta manera, proposiciones.

El primer principio en la elaboración de mapas conceptuales se refiere a la importancia de identificar y seleccionar los conceptos claves, ya que los mapas conceptuales dirigen su atención hacia un reducido número de ideas, las más importantes, en las que debe concentrarse cualquier tarea específica de aprendizaje.

El segundo principio se refiere a que debemos de incluir los supuestos de diferenciación y jerarquización. Esto quiere decir, que los conceptos 0 ideas más generales 0 inclusivas 
deben ocupar la parte superior o ápice del mapa conceptual y que los conceptos más específicos y menos inclusivos, según su grado, van ocupando los demás lugares inferiores, los ejemplos deberán colocarse en la parte más inferior.

El tercer principio se refiere a la necesidad de relacionar los conceptos coherentemente, teniendo un orden lógico. Para lograrlo debemos utilizar adecuadamente las palabras enlace, con ello lograremos construir proposiciones con sentido lógico. Debemos lograr que por medio del mapa conceptual podamos reconocer y reconciliar los nuevos conceptos con los ya aprendidos para poder combinarlos, explorando las relaciones entre ellos.

El cuarto principio es la utilidad del mapa conceptual como instrumento de evaluación, ya sea como diagnóstico, pues nos mostrará lo que el estudiante ya sabe; ya sea durante el desarrollo de un tema, o como actividad final de un tema para medir la adquisición y el grado de asimilación del educando. Para ello podemos trabajar en forma individual o grupal.

El quinto principio se refiere a que los mapas conceptuales deben de construirse y reconstruirse con los demás, lo que constituye un esfuerzo solidario.

Todo lo anterior ayuda a los estudiantes a entender cómo se aprende y el conocimiento sobre el aprendizaje nos sirve para mostrarles como nosotros construimos los nuevos conocimientos.

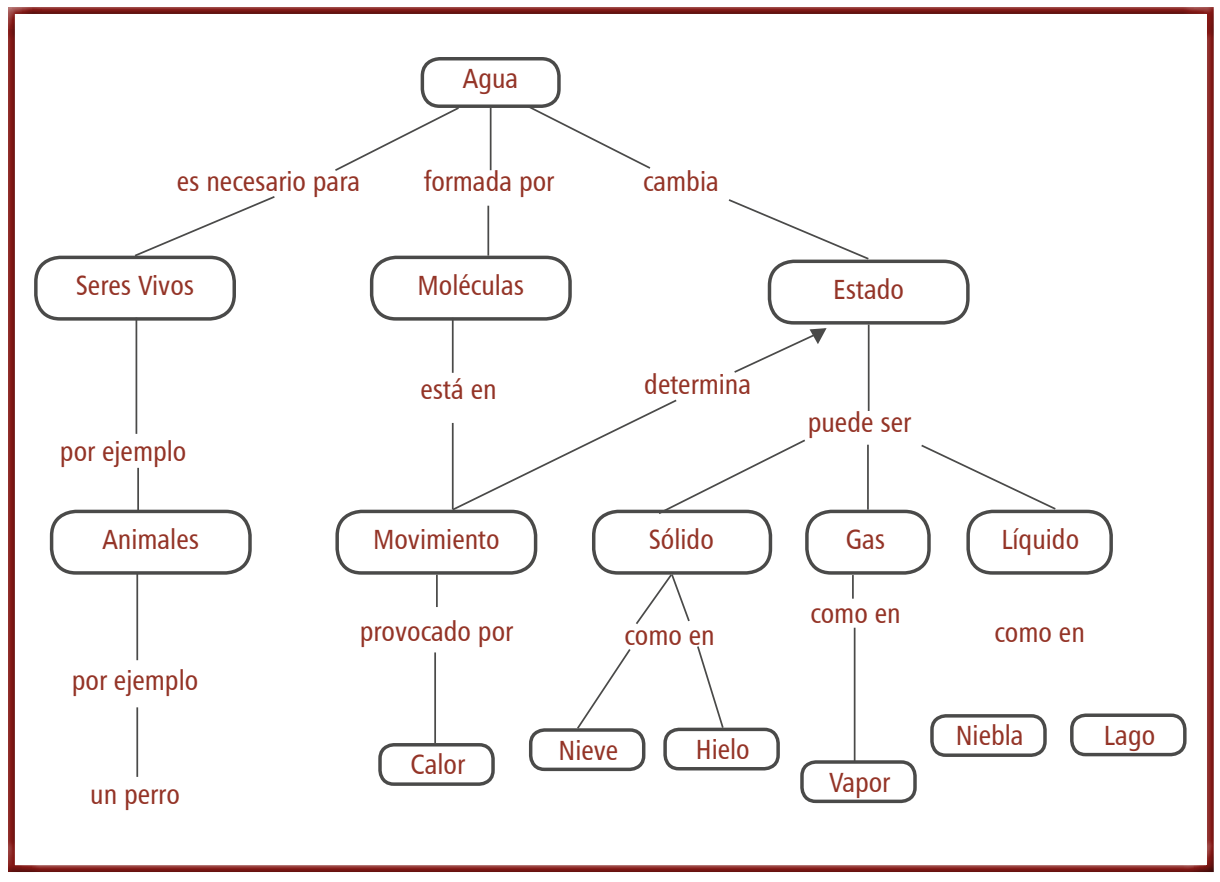




\section{Preparando a los estudiantes para construir mapas conceptuales}

Debemos de tomar en cuenta las siguientes recomendaciones:

a) Ayudar a nuestros estudiantes a reconocer los conceptos claves y sus relaciones, dentro del texto significativo, en forma explícita.

b) Cada uno de nosotros puede imaginar los conceptos, las cosas o acontecimientos, de manera diferente. Es decir que, a pesar de conocer el mismo concepto, cada uno de nosotros puede imaginarlo de varias formas. Por ello, a veces, nos es difícil entendernos mutuamente.

c) Aislar los conceptos y palabras enlace, ya que son importantes para la transmisión de significados.

d) Aprovechar los mapas conceptuales para visualizar las relaciones jerárquicas entre conceptos; su impacto visual es muy importante. Asimismo, debemos aprovecharlos para facilitar nuestro aprendizaje y recuerdo.

e) En la medida de lo posible, los conceptos sólo deben de aparecer una vez.

f) Los mapas conceptuales pueden y deben dibujarse varias veces, ya que el primer mapa conceptual tiene, por lo general, algún defecto.

g) Los mapas conceptuales deben de contener un número reducido de conceptos, lo que favorecerá la claridad.

h) Con los mapas conceptuales los educandos repasan gramática y semántica.

i) Los mapas conceptuales permitirán un aprendizaje rápido y eficiente, es decir, significativo.

\section{Mapas preconceptuales o nocionales}

Tenemos que considerar las siguientes etapas del pensamiento:

1. Pensamiento nocional.

* Edad promedio

* Referentes

* Instrumentos de Conoc.

* Procesos intelectuales

* Productos
: 2 a 6 años

: Hechos

: Nociones

: Proyección - Introyección

: Aseveraciones 
2. Pensamiento conceptual.-

* Edad promedio

* Referentes

* Instrumentos de Conoc.

* Procesos intelectuales

* Productos
: 7 a 11 años

: Aseveraciones

: Conceptos

: Procesos Clasales, relacionales y operacionales

: Proposiciones

3. Pensamiento formal.-

* Edad promedio

: 12 a15 años

* Referentes

: Proposiciones

* Instrumentos de Conoc.

: Conceptos

* Procesos intelectuales

: Razón Interproposicional

* Productos

: Deducciones - Inducciones

4. Pensamiento categorial.-

* Edad promedio

: $>15$ años

* Referentes

: Sistemas proposicionales

* Instrumentos de Conoc.

- Categorías

* Procesos intelectuales

: Métodos de Pensamiento

* Productos

: Microteorías

5. Pensamiento científico.-

* Referentes

: Teorías

* Instrumentos de Conoc.

: Paradigmas

* Procesos intelectuales

: Métodos de Inv. Cient.

* Productos

: Disciplinas

Considerando las etapas del pensamiento humano tendremos que trabajar en el nivel inicial y los primeros años de primaria con el nivel nocional por lo que los mapas que elaboren nuestros estudiantes serán mapas nocionales, cognitivos y/o preconceptuales.

Dentro del objetivo de "enseñar a pensar" los mapas preconceptuales nos servirán para iniciar la creación de estructuras cognitivas de secuenciación y clasificación. 
Asimismo, ya que su nivel de lecto escritura es bajo, tendremos que trabajar al nivel de sus experiencias personales o grupales cotidianas. Por ello, nuestra intervención será mayor para poder guiarlos en la elaboración de sus gráficos y su organización.

Una de las posibilidades de trabajo en clase es dibujar diferentes conceptos relacionados ya sea de un texto o experiencia y comenzar a relacionarlos; se le podría encomendar a los estudiantes en forma individual o grupal. Más adelante, la construcción de los mapas nocionales debe de ser asumida casi en su totalidad por ellos.

Debemos remarcar que la función del maestro es más bien la de facilitador del aprendizaje de sus educandos.

\section{ESTRATEGIAS PARA TRABAJAR MAPAS CONCEPTUALES EN PRIMARIA}

\section{Actividades previas}

1. Presénteles un mapa físico de cualquier país o continente y pregúnteles qué es lo que ellos ven. Luego, explíqueles que los mapas físicos representan países, zonas o regiones del planeta. "Ahora Uds. van a aprender a confeccionar un tipo de mapa: el Mapa Conceptual, es decir, aprenderán a dibujar en una hoja de papel aquellos conocimientos que han adquirido en clases y que están guardados en sus mentes".

2. A continuación, pídales que cierren sus ojos y empiece a mencionarles algunos objetos conocidos por ellos, tales como: carpeta, pizarra, puerta. Pregúnteles si se le viene una imagen a su mente, si su respuesta es afirmativa vaya escribiéndolos en la pizarra, luego, pídales que ellos le den más ejemplos. Paso seguido haga lo mismo, pero mencióneles acontecimientos, tales como: caminar, correr, bailar. Repita lo anterior.

3. Presente la palabra concepto y explique que es la palabra que empleamos para designar cierta "imagen" de un objeto o acontecimiento. A continuación, pregúnteles si todas las palabras escritas en la pizarra les producen imágenes mentales.

4. Paso seguido escriba en las palabras como: la, están, con, entonces, por ejemplo, de, si, entre otras palabras. Pregúnteles si estas palabras les producen imágenes mentales. Ante la respuesta, negativa, debemos de hacerlos reconocer que no son términos conceptuales, sino palabras-enlace que utilizamos para unir términos conceptuales. Enseguida, pídales que les mencionen otras palabras-enlace.

5. Construya oraciones cortas con dos conceptos y una palabra-enlace, trate de utilizar las palabras anotadas en la pizarra. Haga evidente que en nuestro lenguaje escrito y oral usamos términos conceptuales (conceptos) y términos de enlace (palabras enlace). 
6. Pídales a los estudiantes que lean las frases u oraciones escritas en la pizarra. Haga que ellos distingan entre conceptos y palabras enlace.

7. Explique que la mayoría de palabras que aparecen en un diccionario son términos conceptuales.

\section{Elaboración de mapas conceptuales}

1. Prepare una lista de 10 a 12 conceptos conocidos y que estén relacionados. Ordénelos en forma jerárquica de los más generales o inclusivos hasta los más específicos. Estos podrían ser: planetas, estrellas, Vía Láctea, Universo, galaxias, Sol, satélites, Luna, Tierra.

2. Ordenados jerárquicamente del más general al menos inclusivo, tendría el siguiente orden:

Pídales que formen proposiciones con los conceptos seleccionados y jerarquizados.

3. Construya el respectivo mapa conceptual, pidiéndoles su ayuda para incorporar las palabras-enlace.

4. Haga que ellos lean las proposiciones que presenta el mapa conceptual.

5. Pregunte si alguien puede conectar nuevos conceptos en el mapa conceptual. Por ejemplo: planeta azul, Marte, planeta rojo.

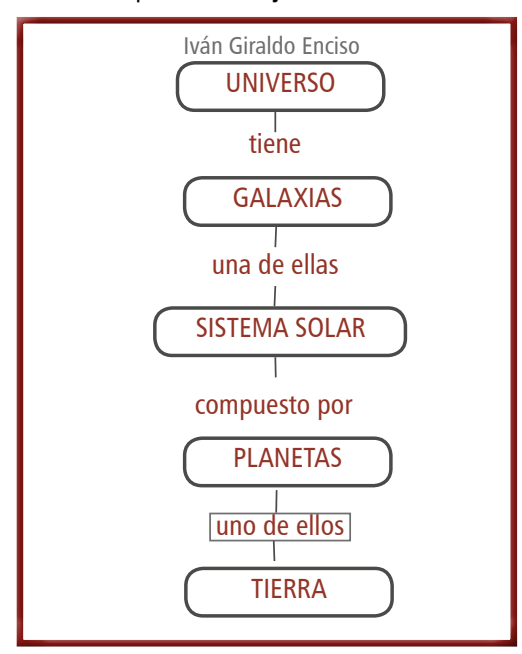

6. Pida que copien el mapa conceptual y que le agreguen los conceptos anteriores con sus respectivas palabras enlace. 
7. Proporcione una nueva lista de conceptos relacionados para que ellos puedan construir sus propios mapas conceptuales: extremidades, cabeza, tronco, nariz, ojos, corazón, pulmones, brazos, piernas, estómago, boca, orejas, riñones, cuerpo humano.

8. Haga que presenten sus mapas conceptuales (en papelógrafo) y que lo expongan. Evite las críticas y resalte los aspectos positivos y el esfuerzo realizado. Acordémonos que esto es un proceso y por ende habrá que retroalimentarlos.

9. Proponga que vuelvan a hacer el mapa conceptual buscando una mejor organización y jerarquización.

10. Elija y reparta una breve narración o parte del material didáctico con el que cuentan, de 10 a 30 oraciones. Enseguida ayúdeles a identificar conceptos y palabras-enlace claves.

11. Pregúnteles cuáles son los conceptos necesarios para poder contar la historia de su material. Pídales que identifiquen los conceptos con un círculo. Luego prepararán una lista ordenada y jerarquizada de los conceptos.

12. Haga que sus estudiantes debatan sobre las listas y su jerarquía. Acto seguido se abocarán a construir sus mapas conceptuales.

13. Ellos deben exponer en clase sus mapas conceptuales. Si cualquiera puede leer y entender la historia descrita en el mapa conceptual, entonces el mapa conceptual cumple con su objetivo. Los docentes debemos evitar las críticas; sus compañeros se encargarán de ello y nosotros, como facilitadores, debemos orientar las críticas.

14. Haga que ellos construyan mapas conceptuales sobre algún tema que ellos conozcan bien o que les guste mucho y que lo presenten en clase. Los mapas conceptuales podrían pegarse en el aula junto con las narraciones y/o narradores para que puedan verlos otras personas; si esto se muestra en una asamblea de padres, sería lo apropiado.

15. A partir de aquí casi todas las actividades de la clase deberían relacionarse con la elaboración de mapas conceptuales, pero también debemos de dosificarla para no saturar a nuestros educandos.

\section{ESTRATEGIAS PARA TRABAJAR MAPAS CONCEPTUALES EN SECUNDARIA}

\section{Actividades previas}

1. Presénteles un mapa físico de cualquier país o continente y pregúnteles qué es lo que ellos ven, luego, explíqueles que los mapas físicos representan países, zonas o regiones del planeta. "Ahora Uds. van a aprender a confeccionar un tipo de mapa: el mapa conceptual; es decir, aprenderán a dibujar en una hoja de papel aquellos conocimientos que han adquirido en clases y que están guardados en sus mentes". 
2. Muéstreles una lista de objetos y otra de acontecimientos que les sean familiares, como carpeta, pizarra, puerta, caminar, correr, bailar. Pregúnteles si son capaces de decir en que se diferencian las dos listas presentadas. Trate de ayudarlos y luego ponga el título a cada lista.

3. Pídales que describan lo que piensan cuando escuchan las palabras carpeta, puerta, pizarra, entre otras. Ayúdelos a que se den cuenta de que, si bien usamos las mismas palabras, cada uno de nosotros puede imaginar las cosas de manera ligeramente distinta. Explíqueles que estas imágenes que vienen a nuestra mente cuando mencionamos algunas palabras, son conceptos.

4. Ahora repita la actividad anterior, pero mencionando acontecimientos como correr, llorar, escribir, etc. Explíqueles que una de las razones por las que a veces no nos entendemos es porque nuestros conceptos nunca son iguales, aunque conozcamos las mismas palabras. Indíqueles que las palabras son signos que designan conceptos, pero cada uno adquiere sus propios significados para las palabras

5. En seguida empiece a nombrar palabras: como, si, entonces, donde, el, la, es, son, etc. Luego, pregúnteles qué es lo que se le viene a la mente cuando oyen cada una de estas palabras. Entonces hágales saber que son las Ilamadas palabras-enlace Estas se usan para unir o enlazar conceptos, formando proposiciones.

6. Pídales que formen frases cortas e identifiquen los conceptos y las palabras-enlace, identificando si los conceptos son objetos o acontecimientos.

7. Elija un texto que transmita un mensaje concreto. Pídales que identifiquen los conceptos principales y que anoten algunas palabras-enlace para el desarrollo del argumento de la narración del mapa conceptual.

\section{Elaboración de mapas conceptuales:}

1. Elija párrafos o textos cortos significativos y haga que ellos lo lean y luego seleccionen los conceptos más importantes. Luego de identificarlos haga una lista de ellos.

2. Identifique el concepto más importante o más inclusivo y jerárquico. Haga una nueva lista, ordenada de mayor a menor generalidad e inclusividad: el concepto más inclusivo debe presidir esta lista.

3. Elabore el mapa conceptual siguiendo la lista ordenada de conceptos. Pida que ellos elijan las palabras-enlace apropiadas.

4. Por lo general, la simetría de estos primeros mapas conceptuales es mala, por ello debemos de rehacerlos. Podemos hacerlos dos o tres veces, si fuera conveniente.

5. Haga que cada uno interprete su mapa conceptual, así lo hayan hecho en forma grupal. 
Si los estudiantes pueden interpretar el mapa conceptual, entonces podríamos decir que el mapa conceptual cumplió su cometido.

6. Evalúe el mapa conceptual. Muéstreles el mapa conceptual de referencia y haga que debatan sobre la evaluación y calificación de sus mapas conceptuales.

7. Señale las posibles enmiendas que tendrán por finalidad un mejor entendimiento del mapa conceptual.

8. Incluya dos o tres preguntas en los próximos exámenes, sobre los mapas conceptuales, pues ellos son un valioso instrumento evaluativo.

\section{Fases para la elaboración de mapas conceptuales}

La elaboración de un mapa conceptual debe comprender las siguientes fases:

10 Trabajar sobre el reconocimiento de conceptos y palabras enlace.

$2^{\circ}$ Analizar el texto:

a) Aplicar la técnica de la lectura comprensiva:

a.1 Lectura rápida, para reconocer el tema tratado.

a.2 Reconocer ideas claves: causas, características, tipos, consecuencias, entre otras.

a.3 Subrayar la idea principal de cada párrafo.

a.4 Reconocer las oraciones complementarias, las que complementan las ideas claves.

$3^{\circ}$ Reconocer conceptos y palabras enlace del texto analizado.

$4^{0}$ Elaboración del mapa conceptual:

a) Hacer el borrador del mapa conceptual.

b) Revisar y reajustar el mapa conceptual.

c) Elaborar el mapa conceptual final.

Debemos de recalcar el tener en cuenta la significatividad lógica y psicológica del texto que se va a trabajar, ya que debe de estar de acuerdo a la edad de nuestros estudiantes y poseer cierta familiaridad con la estructura cognitiva de ellos.

Asimismo, debemos ayudar a nuestros estudiantes a codificar y decodificar las informaciones, estimular el ordenamiento y organización de la información, pues ello favorecerá la interpretación, integración, selección y abstracción de los temas. 


\section{Recomendaciones cuando no se han trabajado las ideas previas de los mapas conceptuales}

- Si no hemos trabajado absolutamente nada y queremos introducir esta técnica podríamos tener en cuenta las siguientes experiencias pedagógicas:

10 Podríamos empezar nuestras clases con una explicación del tema a trabajar, pero haciendo un mapa conceptual mientras vamos explicando el tema.

Nuestros estudiantes se mostrarán sorprendidos por las "bolitas que hace el profesor". Este hecho nos debe dar pie a explicar que lo escrito dentro de las elipses son conceptos o términos conceptuales (que se encuentran dentro de un texto determinado) y las palabras que se encuentran entre las líneas o flechas son las palabras-enlace, que unen los conceptos y le dan sentido.

Podríamos ir más adelante explicando, brevemente, lo que es un concepto: "una palabra que empleamos para designar la imagen de un objeto o acontecimiento que se genera en nuestra mente cuando la mencionan. Asimismo, deberíamos de explicar lo que son las palabras enlace: "son palabras que unen conceptos formando proposiciones, dándoles sentido y coherencia"

$2^{\circ}$ Otra alternativa seria la siguiente: Un educando leería un texto en voz alta, los demás seguirían la lectura. Como facilitadores del aprendizaje, identificaríamos los conceptos más importantes (anotándolos en la pizarra). A continuación, seleccionaríamos los conceptos más importantes y los ordenaríamos por jerarquía, con la activa participación de ellos. El criterio de jerarquización debe de ser de mayor a menor inclusividad.

Paso seguido, les pediríamos que formen proposiciones, utilizando los conceptos seleccionados y jerarquizados. En esta etapa, aún podríamos ayudarlos dándoles ideas sobre la elección de las palabrasenlace más apropiadas.

Finalmente construirían sus mapas conceptuales.

$3^{\circ}$ Una tercera alternativa es cuando nuestros estudiantes tienen algunas nociones sobre los mapas conceptuales.

Luego de haber trabajado la noción de concepto y palabras enlace, pasaremos a entregarles un texto significativo a todos los educandos y les pediremos que identifiquen y seleccionen los principales conceptos.

A continuación, ordenarán los conceptos seleccionados, por jerarquía.

Con los conceptos seleccionados y jerarquizados nuestros estudiantes formarán proposiciones. Ellos le agregarán las palabras-enlace más convenientes.

Finalmente, construirán sus propios mapas conceptuales. 
$4^{0}$ La cuarta alternativa podría ser complementaria a las anteriores; se refiere a presentar un mapa conceptual incompleto para que ellos lo completen.

- Uno de los debates actuales es sobre la conveniencia o inconveniencia del trabajo individual y/o grupal. En el caso de los mapas conceptuales al iniciar esta técnica y en su proceso deberíamos de trabajar en forma grupal; pero una vez que se logra dominarla, se trabajará en forma individual. .

- Asimismo, en nuestros exámenes deberíamos de pedir que construyan un mapa conceptual.

\section{Evaluación de los mapas conceptuales}

Tengamos en cuenta los siguientes criterios:

1. Proposiciones.- En un mapa conceptual la relación entre los conceptos debe de ser significativa y válida, por lo que debe tenerse muy en cuenta. Además debemos evaluar si la palabra-enlace cumple con relacionar coherentemente los términos conceptuales.

2. Jerarquía.- El mapa conceptual debe guardar la jerarquía del texto en cuestión, considerando que un concepto más inclusor puede estar subordinado a otro de menor inclusividad, dependiendo del contexto en el que se haya el concepto aludido. Además, debemos de tener en cuenta que el mapa conceptual debe corresponder al texto analizado.

3. Relaciones o Conexiones Cruzadas.- Cuando se inserta una relación o conexión cruzada significativa se demuestra una gran capacidad creativa. Por ello debemos de identificar, reconocer y gratificar, de manera especial, estas relaciones y/o conexiones.

4. Ejemplos.- Estos deben de ocupar el último lugar en los mapas conceptuales, por lo que debemos de tener en cuenta si son válidos, adecuados o no.

5. Mapa conceptual Paradigma.- Podríamos construir un mapa conceptual referencial y asignarle una puntuación a cada criterio anotado. Sin embargo, nuestros estudiantes podrían construir mejores mapas conceptuales que el referencial, por lo que debemos de reconsiderar nuestra puntuación.

- Debemos de reconocer la creatividad de nuestros educandos, en esta perspectiva ellos podrían alterar el orden jerárquico y no por ello su mapa conceptual sería inválido.

- Una vez que ellos dominen la jerarquía podríamos obviar algunos criterios y asumir que el criterio máximo es que, si el mapa conceptual lo puede leer cualquiera, entonces el mapa conceptual ha cumplido su cometido y por ende está bien hecho. 
6. La traducción numérica es una calificación concreta que la tenemos que realizar nosotros. Pero hay algunas circunstancias que influyen en los criterios evaluativos:

- La regularidad en el uso del mapa conceptual en el aula, en este caso, si no hemos trabajado regularmente esta técnica no podríamos pedir grandes resultados.

- Cómo se ha utilizado: como eje de ejecución, técnica de estudio, síntesis, entre otras.

- El trabajo de otras técnicas metodológicas.

7. Esta técnica ayuda a trabajar valores sociales como: participación, solidaridad, consenso, diálogo, tolerancia; y valores personales como: autoestima, capacidad crítica y reflexiva. Todos ellos deberían de ser evaluados.

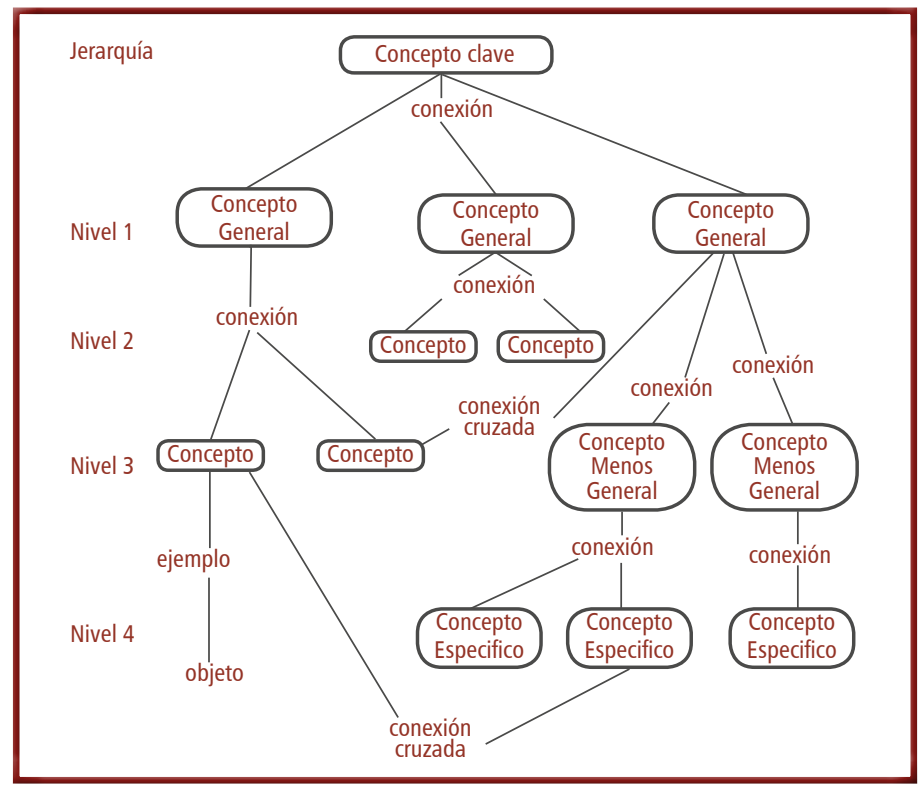

\section{Algunas objeciones a los mapas conceptuales}

A través del trabajo y utilización de los mapas conceptuales en el profesorado se han dado diversas dudas e interrogantes sobre ellos; aquí presentamos algunas de ellas:

La duda más difundida es saber si se aplica a todas las asignaturas o no. Debemos saber que Novak aplica los mapas conceptuales a las Ciencias Naturales; es Ontoria quien aplica los mapas conceptuales a las demás ciencias, si bien es cierto que los mapas conceptuales se prestaban más al campo científico, pues sus conceptos son más aceptados mientras que las demás ciencias la significación, delimitación y convencionalismo de los conceptos son más problemáticos. 
Entonces las ciencias como: física, química, biología, matemática, entre otras, su lenguaje formal, es por ende, más estructurado y uniforme, por lo que la comprensión y significado de sus conceptos son aceptados por la totalidad de la comunidad científica. Lo contrario acontece con las ciencias humanas: filosofía, historia, sociología, lingüística, entre otras; en ellas la comprensión y significado de los conceptos tienden a ser más problemático, pues no todos los miembros de estas comunidades están de acuerdo con los significados atribuidos a los conceptos. Pero la desventaja de la heterogeneidad del significado se convierte en ventaja para desarrollar un pensamiento divergente, creativo y autónomo.

La segunda objeción con la que nos encontramos es acerca de que los mapas conceptuales requieren mayor trabajo y tiempo lo que les impediría cumplir con los programas o unidades de aprendizaje. En nuestra práctica diaria hemos podido comprobar que su utilización ahorra tiempo, si bien al introducir esta técnica supone un desgaste de tiempo, este se recupera con creces una vez que nuestros estudiantes empiecen a dominarla. Es más, ellos testimonian que estudian mejor, que los conocimientos se les queda mejor impregnados, con lo cual el repaso es más rápido y ágil llegando a comprender mejor los temas estudiados. Debe de tomarse atención sobre la frecuencia del uso de esta técnica, pues si no se utiliza con regularidad, su dominio y capacidad de comprensión producirán efectos contrarios.

En este caso concluiremos que lo que perdamos en extensión lo ganaremos en profundidad.

Otra objeción frecuente es afirmar que los mapas conceptuales empobrecen la expresión hablada y escrita. Si vemos un mapa conceptual cualquiera nos daría la idea de que es muy conciso, pero el mapa conceptual nos ayuda a fijar conceptos claves relacionados con palabras enlace que nos ayudarán a darles sentido por medio del discurso que puede ser muchas veces amplio.

La característica de tener impacto visual trae consigo la siguiente objeción: que se apoya en el estilo visual de aprendizaje. Si bien el mapa conceptual remarca el predominio del estilo visual de aprendizaje, debemos de decir que no hay estilos de aprendizaje puros. También habría que aclarar que los estilos auditivo y kinestésico se involucran muy bien con los mapas conceptuales, pues estos se trabajan, mayoritariamente, en forma grupal.

Cuando una técnica se pone "de moda" los que la utilizan la sobrestiman llegando a sostener que es la panacea del aprendizaje. Debemos de entender que, si bien es una técnica que tiene grandes bondades y además es lúdica, no debemos cometer el error de circunscribirnos solo a ella sin tomar en cuenta las otras técnicas de estudio. Debemos de dosificar su uso para no saturarlos. No debemos olvidar que hay otras técnicas válidas de aprendizaje y estudio. 
Hace algunos años Antonio Ontoria y Ana Molina en su libro "Los Mapas Conceptuales y su Aplicación en el Aula" presentaron una encuesta aplicada por ellos:

$1^{\text {a }}$ pregunta: ¿Se ha entendido la técnica del mapa conceptual?

- Es una representación gráfica de conceptos.

- Ayuda a seleccionar los elementos fundamentales.

- Es fácil de memorizar y de estudiar.

- Es una forma original de estudiar

- Se ve más claro

- Están todos los conceptos principales y, aunque está muy simplificado, solo con desarrollarlo un poco, lo entiendo.

$2^{\mathrm{a}}$ pregunta: ¿te resulta fácil aplicarla?

- Se memoriza y se entiende mejor.

- Se sacan las ideas generales del tema.

- Se entera uno mejor.

- Le quita menos tiempo.

- Es más ameno y menos complicado.

- Es más entretenido.

- Nos ayuda a estudiar.

$3^{\text {a }}$ pregunta: ¿Qué ventajas te da?

- Entiendo mejor el tema.

- Lo recuerdo mejor.

- Trabajo con más gusto.

- Se tarda menos tiempo en estudiar.

- Sirve para reforzar.

- Se sacan las ideas básicas.

$4^{\mathrm{a}}$ pregunta: ¿Te gusta su uso por el profesor?

- Es más rápido.

- Su visión es global. 
- Se queda mejor, se entiende mejor.

- No aburre, es más cómodo.

- Se estudia con más gusto.

- Es muy útil para repasar.

- Se memoriza mejor.

- La clase se hace más llevadera.

En nuestro caso hemos recibido testimonios de algunos profesores que sólo teniendo las ideas básicas de los mapas conceptuales se atrevieron a utilizarlo en clase y su experiencia es muy gratificante. Pensemos, entonces, si dominamos la técnica, lo valioso que será para nuestro trabajo diario en las aulas.

\section{Tesina}

\section{Investigación sobre la aplicación de los mapas conceptuales \\ Diseño de investigación}

Problema científico:

¿Cómo contribuir a que los estudiantes del $9^{\circ}$ "A" del colegio Champagnat de Miraflores identifiquen adecuadamente los elementos fundamentales de los mapas conceptuales?

Objeto de investigación:

Mapas conceptuales

Campo de estudio:

Elementos fundamentales de los mapas conceptuales.

Objetivo:

Identificar los elementos fundamentales de los mapas conceptuales.

Preguntas científicas:

1. ¿Cuál es el nivel inicial de los estudiantes sobre la elaboración de mapas conceptuales?

2. ¿Cuáles son los fundamentos teóricos de los mapas conceptuales? 
3. ¿Cuáles son los elementos fundamentales de los mapas conceptuales?

4. ¿Cuáles son las características de los mapas conceptuales?

5. ¿Cuáles son los principios metodológicos de los mapas conceptuales?

Tareas:

1. Diagnóstico del nivel inicial en la elaboración de mapas conceptuales.

2. Sistematización de los fundamentos teóricos sobre los mapas conceptuales.

3. Identificación de los elementos fundamentales de los mapas conceptuales.

4. Caracterización de los mapas conceptuales.

5. Identificación de los principios metodológicos para la elaboración de los mapas conceptuales.

Variable:

Mapa conceptual

\section{Diseño metodológico}

Unidad de estudio:

Consideramos como población a los 36 estudiantes del $9^{\circ}$ "A" del Colegio Champagnat de Miraflores.

Decisión muestral:

La muestra se obtendrá mediante la técnica probabilística estratificada: 25\%

Métodos a utilizar:

- Teóricos:

Análisis - síntesis

Inducción - deducción

- Empíricos:

Encuesta

Resumen

Nuestros estudiantes confunden los mapas conceptuales con esquemas - resúmenes, cuadros sinópticos, ideas principales, entre otros. Por ello la necesidad de identificar y reconocer los elementos fundamentales de los mapas conceptuales. 
La noción de mapas conceptuales se desarrolla en la universidad de Cornell, en los 60, supervisado por Joseph Novak.

Asume la noción de los esquemas conceptuales de Piaget y busca desarrollar el aprendizaje significativo de Ausubel. Novak considera que los mapas conceptuales son una herramienta de trabajo que permite la confrontación y análisis de la forma de pensar de los estudiantes.

El elemento central de los mapas conceptuales son los conceptos, que vendrían a ser la palabra que se emplea para designar la imagen de un objeto o acontecimiento en la mente. Estos conceptos unidos por las palabras enlace forman relaciones significativas o proposiciones. Mientras que el concepto debe de pertenecer al texto estudiado la palabra enlace puede ser inventada.

Entre los elementos están los signos gráficos: las elipses que engloban los conceptos y las líneas que unen los conceptos y donde van las palabras - enlace.

Muchas personas creen que los mapas conceptuales no son apropiados para los cursos de ciencias, sin embargo, estos fueron utilizados para ciencias naturales, por lo tanto, su utilidad se da en todas las áreas académicas.

Todo mapa conceptual se distingue por las siguientes características:

Jerarquía, Selección e Impacto Visual.

Esperamos que este trabajo despierte el interés por trabajar adecuadamente los mapas conceptuales.

\section{Anexos}

Encuesta:

1. De las técnicas utilizadas a lo largo del año, ¿cuál te ha sido más provechosa para tus estudios?
a) Mapas conceptuales
b) Mapas mentales
c) Línea del tiempo
d) Historieta
e) Otro

2. ¿Cuál ha sido más fácil de aprender?
a) Mapas conceptuales
b) Mapas mentales
c) Línea del tiempo
d) Historieta
e) Otro 
3. ¿Cuál se ajusta a tu estilo?
a) Mapas conceptuales
b) Mapas mentales
c) Línea del tiempo
d) Historieta
e) Otro

4. ¿En qué grado empezaste a trabajar los mapas conceptuales
a) séptimo
b) octavo
c) noveno
d) En primaria (especifica)

5. ¿Utilizabas los mapas conceptuales?
a) Alguna vez
b) Nunca
c) Siempre

6. ¿Utilizas los mapas conceptuales para estudiar?
a) Alguna vez
b) Nunca
c) Siempre

7. ¿Utilizarás los mapas conceptuales en adelante?
a) Alguna vez
b) Nunca
c) Siempre

8. ¿Qué son los mapas conceptuales?
a) Una técnica para reconocer los conceptos y palabras-enlace.
b) Un esquema donde se identifican las ideas principales.
c) Un organigrama.
d) Un resumen ordenado en cuadros.
e) Otro

9. ¿Cuál es la principal dificultad para elaborar los mapas conceptuales?
a) Es difícil de identificar los conceptos.
b) Es difícil dejar de lado varios conceptos que estén en uno.
c) Si el texto es muy largo no sé cómo reducir los conceptos.
d) Es difícil ordenar los conceptos y las palabras-enlace.
e) Otro

10. ¿Los mapas conceptuales te ayudaron a resolver algún examen o prueba?
b) Alguna vez
b) Nunca
c) siempre 
11. ¿Qué ventajas tienen los mapas conceptuales?

a) Me ayudan a estudiar mejor.

b) Me ordenan las ideas de lo que estoy estudiando.

c) Es menos complicado y más entretenido.

d) Recuerdo mejor lo que estudio.

e) Se memoriza y entiende mejor.

\section{Resultados estadísticos}

1. Técnica más utilizada

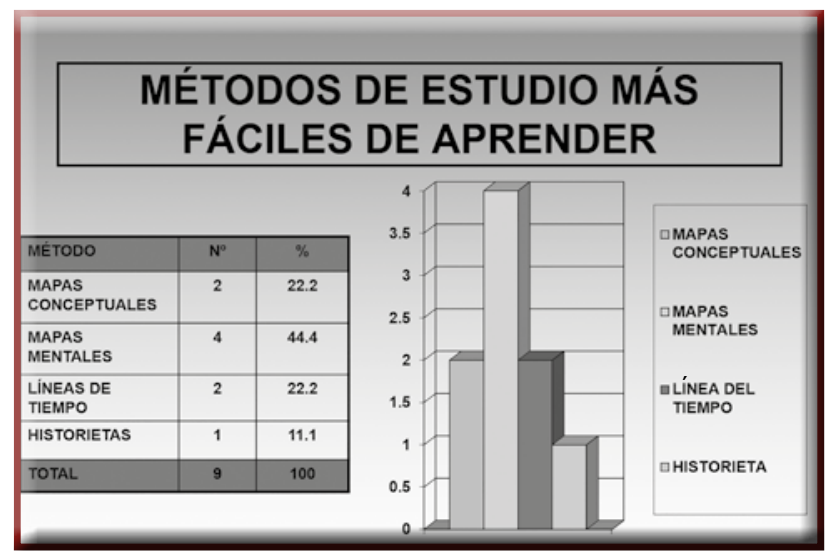

2.

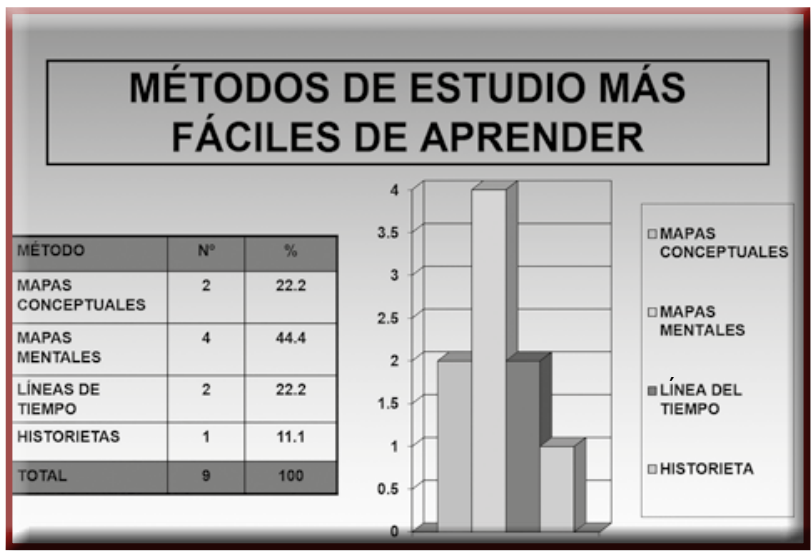


3.

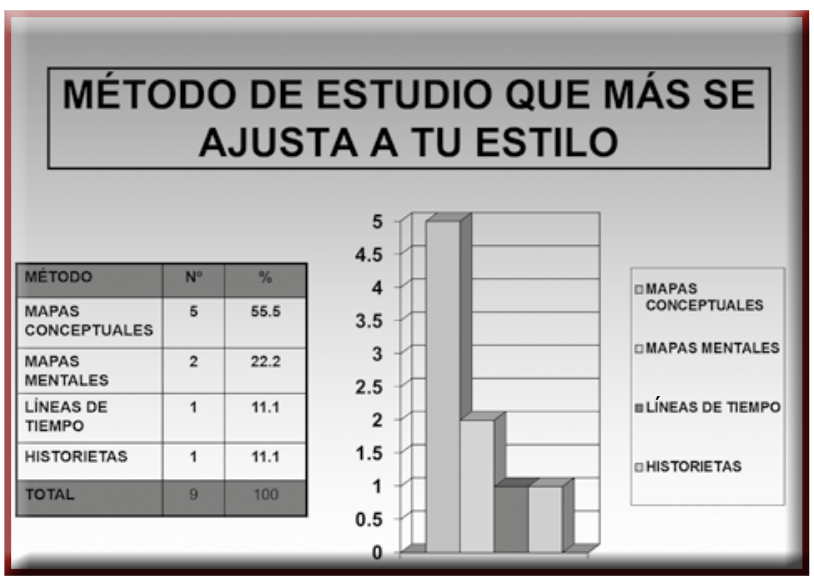

4.

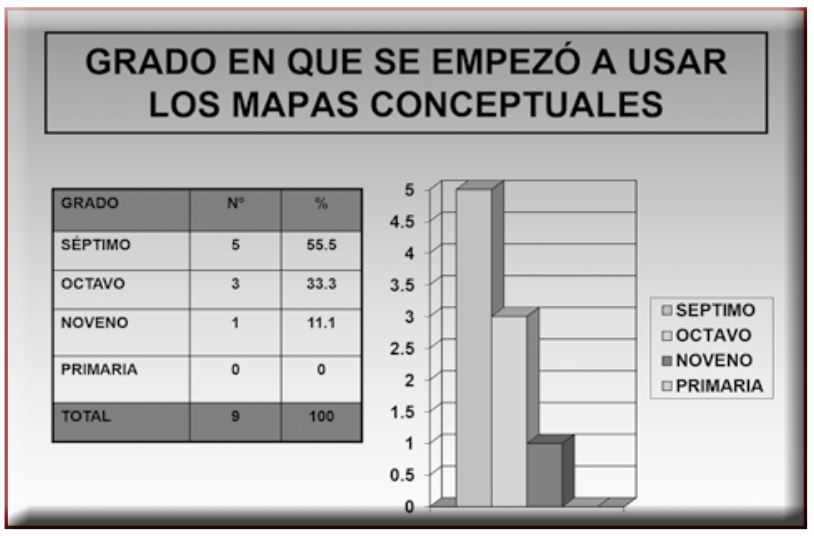

5.

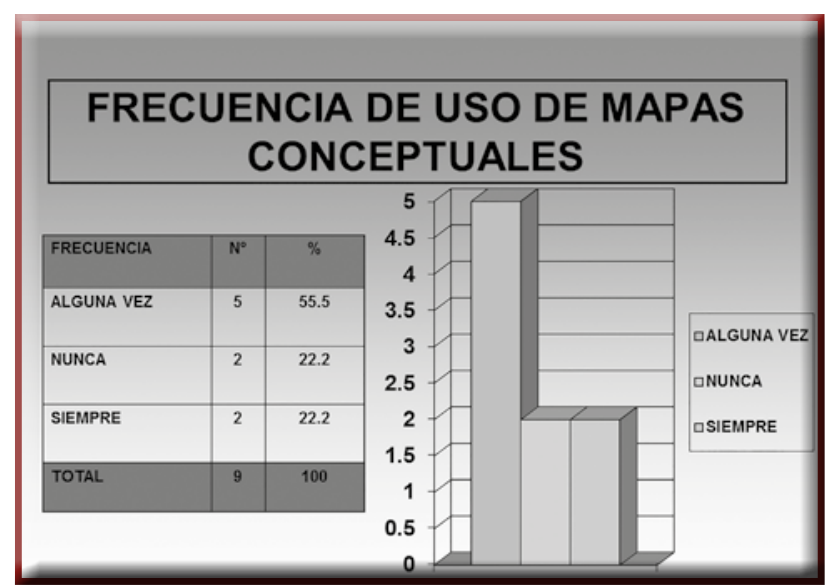


6.

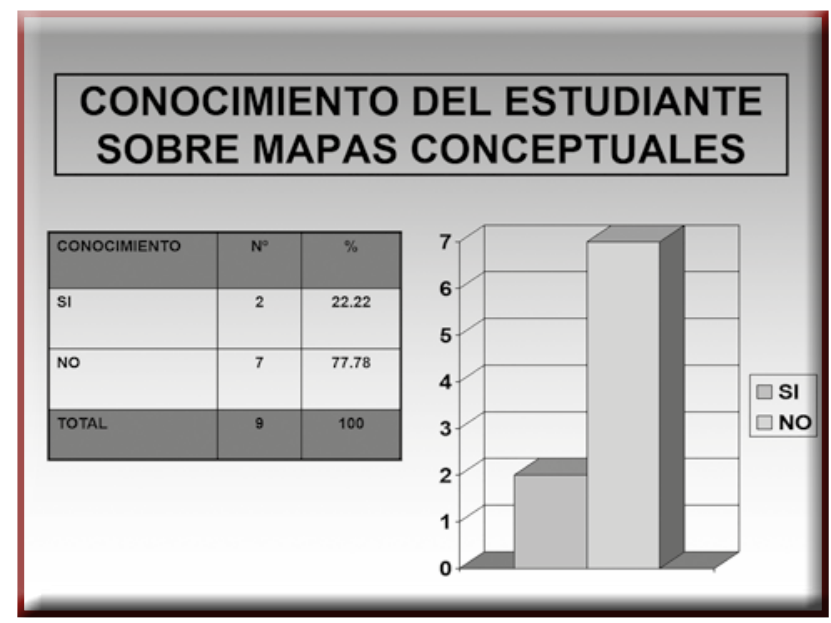

7.

PRINCIPALES DIFICULTADES PARA ELABORAR MAPAS CONCEPTUALES

\begin{tabular}{|c|c|c|c|c|c|}
\hline DIFICULTADES & $\overline{N^{\circ}}$ & $\%$ & \multirow{6}{*}{\multicolumn{2}{|c|}{$\begin{array}{r}2.5 \\
2 \\
1.5\end{array}$}} & \multirow{3}{*}{$\begin{array}{l}\text { 口IDENTIF. } \\
\text { CONCEPTOS } \\
\text { पDEJAR } \\
\text { CONCEPTOS }\end{array}$} \\
\hline $\begin{array}{l}\text { IDENTIFICAR } \\
\text { CONCEPTOS }\end{array}$ & 2 & 22.2 & & & \\
\hline $\begin{array}{l}\text { DEJAR DE LADO } \\
\text { CONCEPTOS QUE } \\
\text { ESTAN EN UNO }\end{array}$ & 3 & 33.3 & & & \\
\hline $\begin{array}{l}\text { REDUCIR LOS } \\
\text { CONCEPTOS DE } \\
\text { TEXTOS LARGOS }\end{array}$ & 3 & 33.3 & & & $\begin{array}{l}\text { 口REDUCIR } \\
\text { CONCEPTOS }\end{array}$ \\
\hline $\begin{array}{l}\text { ORDENAR } \\
\text { CONCEPTOSY } \\
\text { PALABRAS-ENLACE }\end{array}$ & 2 & 22.2 & & & $\begin{array}{l}\text { IORDENAR } \\
\text { CONCEPTOS }\end{array}$ \\
\hline TOTAL & 9 & 100 & & & \\
\hline
\end{tabular}




\section{Referencias}

Arellano, J. y Santoyo, M. (2009); Investigar con mapas conceptuales. Madrid-España

Buzan, Tony (2004); El libro de los mapas mentales. Ed. Urano,

Heimlich, Joan y Pittelman, Susan; (1999); Los mapas semánticos: estrategias de aplicación en el aula. Ed. Visor,

Maya, A. y Díaz, N. (2002); Mapas conceptuales. Colombia

Novak, Joseph D.; (2001); Conocimiento y aprendizaje: los mapas conceptuales como herramientas facilitadoras para escuelas y empresas. Alianza Editorial.

Novak, Joseph D. (1992); Teoría y práctica de la educación. Madrid-España.

Novak, Joseph D. (1998); Conocimiento y aprendizaje. Madrid-España.

Novak, Joseph D. y Gowin, D.B. (1988); Aprendiendo a aprender; Ediciones Martínez Roca, S.A., Barcelona.

Novak, J. D. y Gonzales, F. (1993); Aprendizaje significativo: técnicas y aplicaciones. Argentina

Ontoria, Antonio y Otros (1994); Mapas conceptuales: una técnica para aprender; Ed. Narcea. Madrid.

Ontoria, Antonio y Otros (1996); Mapas conceptuales en el aula. Buenos Aires: Ed. Magisterio del Río de la Plata.

Ontoria, A., Gómez, J. P. R. y Molina, A. (2000); Potenciar la capacidad de aprender a pensar. Madrid-España.

Pérez, C. M. (1997); Constructivismo: una red de innovación pedagógica. Lima-Perú.

Eduteka: Entrevista a Novak, J. del Origen de los Mapas Conceptuales.

http://www.eduteka.org/modulos. php?catx $=4 \&$ idSubX $=90 \& i d a=543 \&$ art $=1$

WEBGRAFIA: Recursos en internet

http://www.conceptmaps.it/KM-LearningTech-esp.htm

http://www.javeriana.edu.co/decisiones/mapas.html 
http://www.netdidactica.com/articulos/mapas.htm

http://es.geocities.com/naolig/tesauros-mapas-conceptuales-topic-maps.htm http://www.eduteka.org/pdfdir/MapasConceptuales.pdf http://www.geocities.com/Area51/Stargate/4295/demc/b3.html http://www.indexnet.santillana.es/mapasConceptos/mapasConceptos.htm 